\title{
Phage therapy
}

\section{Should bacterial resistance to phages be a concern, even in the long run?}

\begin{abstract}
Anni-Maria Örmälä ${ }^{1}$ and Matti Jalasvuori ${ }^{2,3, *}$
${ }^{1}$ Department of Biosciences; University of Helsinki; Helsinki, Finland; ${ }^{2}$ Center of Excellence in Biological Interactions; Department of Biological and Environmental Science; University of Jyväskylä; Jyväskylä, Finland; ${ }^{3}$ Division of Ecology, Evolution and Genetics; Research School of Biology;

Australian National University; Canberra, Australia
\end{abstract}

Keywords: evolution, ecology,

phage-therapy, antibiotic resistance, phage resistance

Submitted: 06/29/12

Revised: 03/06/13

Accepted: 03/06/13

Citation: Örmälä A.-M., Jalasvuori M. ; Phage therapy: Should bacterial resistance to phages be a concern, even in the long run? Bacteriophage 2013; 3:e24219; http://dx.doi.org/10.4161/bact.24219

${ }^{*}$ Correspondence to: Matti Jalasvuori;

Email: matti.jalasvuori@jyu.fi
Bacteriophage therapy, the use of viruses that infect bacteria as antimicrobials, has been championed as a promising alternative to conventional antibiotics. Although in the laboratory bacterial resistance against phages arises rapidly, resistance so far has been an only minor problem for the effectiveness of phage therapy. Resistance to antibiotics, however, has become a major issue after decades of extensive use. Should we expect similar problems after long-term use of phages as antimicrobials? Like antibiotics, phages are often noted to be drivers of bacterial evolution. Should we expect phage-treated pathogens to develop a general resistance to phages over time, a resistance against which only, for example, hypothetically co-evolved phages might be infective? Here we argue that the global infection patterns of phages suggest that this is not necessarily a concern as environmental phages often can infect bacteria with which those phages lack any recent co-evolutionary history.

Bacterial pathogens have been treated effectively with antibiotics for several decades. However, the extensive use of antibiotics, both properly and improperly, has generated strong selection pressure for bacteria to become resistant. ${ }^{1}$ Antibiotic resistant strains are becoming increasingly more common and thus infections acquired especially within hospital settings often are untreatable with conventional therapies. Bacteria typically need only few genes to become resistant to commonly used antibiotics. These resistancegenes often move horizontally between bacteria within selfish genetic elements, such as conjugative plasmids, ${ }^{2}$ and a single element can confer resistance to many different antibiotics. ${ }^{3}$ Furthermore, plasmids spread and persist within populations even in absence of antibiotics, suggesting that decrease in the use of antibiotics is not likely to help the situation. ${ }^{4}$ Although there may be means to get rid of the resistance or prevent its spread, ${ }^{3}$ more generally effective alternatives to conventional antibiotics would be useful for combatting those bacterial pathogens that present as antibiotic resistant.

The ability of bacteriophages to clear and kill bacteria often inspires the idea that these bacterial viruses may be included among these possible alternatives to conventional antibiotic agents, that is, as selectively toxic antibacterials. This initial excitement, however, can fade when it transpires that bacteria also can rapidly develop resistance to phages, even within relatively small bacterial populations (of, e.g., about $10^{8}$ cells). ${ }^{5}$ Bacteria can become resistant by several mechanisms, including for example alteration of receptors to which phages attach or the development of adaptive immunity via interfering CRISPR sequences. ${ }^{6}$ Although mechanisms of bacterial resistance to phages differ substantially from those of chemical antibiotics, the overall result from the perspective of therapy is the same: bacterial proliferation can no longer be controlled by therapeutic agents. Noting the rapidly emerging resistance, the advocates of phage therapy usually bring up the benefits of phage cocktails, in which many different types of phages infect the same species 
or strains, and thus make the emergence of a resistant bacterial cell substantially less likely. ${ }^{7,8}$ Yet, there remains a lingering concern that extensive use of phages will select in one way or another for bacterial strains that are resistant to phage-cocktails. As Krylov et al. has recently argued, it might be more reasonable to use only a single lytic phage, one very specific to the pathogen causing an infection, in order to prevent the emergence of bacteria that are resistant to the valuable broad-host range cocktails. ' Perhaps equivalently to chemical antibiotics, after decades of use of phages numerous bacterial pathogens may be resistant to various phage-cocktails, resulting in major difficulties in treating, e.g., hospital acquired (nosocomial) infections. ${ }^{10}$

Phages are often stated to drive bacterial evolution, ${ }^{11}$ with phages forcing adaptations within bacterial populations, especially phage resistance. Generally phages have a narrow host range and this is so to such an extent that the specificity of a set of different phages has been routinely used to distinguish closely related bacterial strains from one another. ${ }^{12}$ Given the narrow host range, the continuous selection for resistance and the often mentioned evolutionary arms race between bacteria and phages, ${ }^{13,14}$ might we suspect difficulties in future attempts to isolate phages that are effective against potentially multi-phage-resistant bacterial strains and may this compromise the long-term viability of phage therapy? It is specifically this latter question that we address in this commentary.

Phage impact on bacterial evolution can occur in the course of antagonistic co-evolutionary cycles. ${ }^{15,16}$ In these cycles, viruses evolve to re-infect hosts that have already become resistant to earlier types of the same virus. ${ }^{13,14}$ It has been shown moreover that the frequency of bacterial reversion back to phage-susceptible phenotypes is relatively low. ${ }^{17}$ Surface components of bacterial cells in particular evolve rapidly, which likely is due to the fact that they are utilized by phages for host attachment (though also, for pathogens, could be a consequence of immune system evasion). Co-evolutionary arms races between bacteria and their lytic viruses also have been shown to accelerate the rate of molecular evolution of the coevolving species even in laboratory conditions. ${ }^{11,18,19}$ Although not all viruses seem to be able to adapt to infect resistant hosts ${ }^{16,20}$ this scenario nonetheless gives the impression that at least some phages can remain infective only by constantly evolving in terms of their host range. ${ }^{16}$ If pre-defined phage cocktails were used globally and continuously in a manner similar to that of antibiotics, resulting in bacterial evolution but not antimicrobial evolution, then it is possible that phages could with time become 'over-evolved' by their hosts.

Evolution of bacterial resistance to phages, may occur more slowly given use of phage cocktails rather than individual phages, but nevertheless only delay the evolution of bacterial superbugs that are highly resistant, generally, to phages. Alternatively, to the extent that broad bacterial resistance to phages can evolve, cocktails by supplying multiple phages simultaneously could select precisely for such hypothetically broadly phage-resistant bacteria. In any case, this concern, no matter how hypothetical, we feel is a reasonable interpretation of the more general concern that phage therapy may suffer the same fate of antibiotic therapy: the evolution of superbugs for which it is difficult to identify new antibacterials against which resistance is not already present. Alternatively, it could be argued that perhaps only co-evolved phages that have responded evolutionarily in course of ongoing bacterial evolution to phage resistance may remain infective against these hospital specific strains, that is, phages responding mutation by mutation rather than simultaneously to large numbers of anti-phage resistance alleles that have accumulated in target bacteria.

Despite of the above mentioned observations on bacterial evolution, several studies are suggestive that evolution of phage-resistant superbugs is not going to occur. First, the cycles of viral adaptation to evolved hosts and vice versa have been experimentally shown in laboratory conditions and natural habitats. Gomez and Buckling (2011) in particular demonstrated that bacteria in soil were more resistant to their contemporary phages than to past or future phages. ${ }^{21}$ Host resistance to phage infection was thus only a temporary trait of the bacterium. Similarly, viruses were less infective to current bacteria than future or past hosts, suggesting that virus predation was not able to induce changes that would allow only the coevolved viruses to remain infective to their hosts. This, in turn, suggests that viral selection is not forcing genetic divergence that would also prevent the replication of other viruses in any particular host. Phage resistance in many cases can be costly for the bacterium $^{22,23}$ and it thus appears to be disadvantageous for the bacteria to remain resistant against bacteriophages that are no longer present in their local environment. ${ }^{21}$ Moreover, phage-resistant bacteria often lack important surface features that are responsible for bacterial virulence. ${ }^{24,25}$ Resistant bacteria, even if they are not killed by phages, therefore can become mostly harmless. However, sometimes there has been no observable costs associated with resistance, ${ }^{20,26,27}$ demonstrating that resistant phenotypes, at least under certain conditions, may persist.

Even if continuous use of phages forced a bacterial population to become permanently resistant to specific phagecocktails, biogeography studies of phage infection patterns suggest that new infectious phages will nevertheless be available. It has been shown that regardless of the absence of recent contact, phages have remained infective to bacterial host cells on the other side of the world. This is despite the fact that most bacteria are thought to be under constant evolutionary arms race with the phages in their present environment. ${ }^{11}$ For example, metaanalysis of host-phage interactions of 38 separate studies performed by Flores et al. concluded that phages can often infect many different host strains from different origins. ${ }^{28}$ They pointed out that infection patterns were nested, indicating that the 'hard to infect' hosts were infected by generalist phages and not by selective specialists. Correspondingly, Wolf et al. showed in 2003 that they were able to isolate bacteriophages from distant geographical locations for a single freshwater bacterial host (Sphingomonas sp) isolated in $1985 .{ }^{29}$ One of us recently isolated phages from Finland for bacterial strains that were originally collected from Britain in the sixties. These and other examples have demonstrated 
that a single bacteriophage can remain infective to bacteria around the world and that a single bacterium can serve as a host for bacteriophages derived from multiple distant geographical locations. While continuous artificial selection in hospital settings against an over-presented set of phages may still have unpredictable outcomes, evolution in natural communities do not seem to be driving bacteria locally to diversify in such manner that would generally render all other phages without recent co-evolutionary history ineffective. The same is true for archaeal extremophiles dwelling in relatively isolated habitats and their viruses, indicating that the 'global infectivity' is not an evolutionary trend that is restricted to bacteria alone. ${ }^{30}$

Altogether this implies that despite of extensive use of therapeutic phages, a situation where new infectious phages for pathogenic bacteria can no longer be found is unlikely. In a historical perspective, phage therapy has already failed once (as reviewed by Sulakvelidze et al. ${ }^{31}$ ). We need to be careful with our second try, but unnecessary pessimism is unwarranted. Thus, whereas new 'conventional' antibiotics have become increasingly more difficult to develop or discover, current evolutionary as well as ecological understanding of phage biology instead is suggestive of optimism in this regard, with reasonable expectation that new, effective environmental phages will be continually available indefinitely against most bacterial targets.

Disclosure of Potential Conflicts of Interest No potential conflicts of interest were disclosed.

\section{Acknowledgments}

We are grateful to one of the reviewers for his or her valuable help in editing and improving this commentary.

\section{References}

1. Andersson DI, Hughes D. Evolution of antibiotic resistance at non-lethal drug concentrations. Drug Resist Updat 2012; 15:162-72; PMID:22516308; http://dx.doi.org/10.1016/j.drup.2012.03.005

2. Bennett PM. Plasmid encoded antibiotic resistance: acquisition and transfer of antibiotic resistance genes in bacteria. Br J Pharmacol 2008; 153 Suppl 1:S34757; PMID: 18193080

3. Jalasvuori M, Friman VP, Nieminen A, Bamford JK, Buckling A. Bacteriophage selection against a plasmid-encoded sex apparatus leads to the loss of antibiotic-resistance plasmids. Biol Lett 2011; 7:9025; PMID:21632619; http://dx.doi.org/10.1098/ rsbl.2011.0384

4. Dahlberg C, Chao L. Amelioration of the cost of conjugative plasmid carriage in Eschericha coli K12. Genetics 2003; 165:1641-9; PMID:14704155.

5. Lenski RE. Two-step resistance by Escherichia coli B to bacteriophage T2. Genetics 1984; 107:1-7; PMID:6373494

6. Hyman P, Abedon ST. Bacteriophage host range and bacterial resistance. Adv Appl Microbiol 2010; 70:217-48; PMID:20359459; http://dx.doi. org/10.1016/S0065-2164(10)70007-1

7. Gu J, Liu X, Li Y, Han W, Lei L, Yang Y, et al. A method for generation phage cocktail with great therapeutic potential. PLoS One 2012; 7:e31698; PMID:22396736; http://dx.doi.org/10.1371/journal.pone. 0031698

8. Chan BK, Abedon ST. Phage therapy pharmacology phage cocktails. Adv Appl Microbiol 2012; 78:1-23 PMID:22305091; http://dx.doi.org/10.1016/B9780-12-394805-2.00001-4

9. Krylov V, Shaburova O, Krylov S, Pleteneva E. A genetic approach to the development of new therapeutic phages to fight pseudomonas aeruginosa in wound infections. Viruses 2013; 5:15-53; PMID:23420928; http://dx.doi.org/10.3390/v5010015

10. Bush K, Courvalin P, Dantas G, Davies J, Eisenstein B, Huovinen P, et al. Tackling antibiotic resistance. Nat Rev Microbiol 2011; 9:894-6; PMID:22048738; http://dx.doi.org/10.1038/nrmicro2693

11. Clokie MR, Millard AD, Letarov AV, Heaphy S. Phages in nature. Bacteriophage 2011; 1:3145; PMID:21687533; http://dx.doi.org/10.4161/ bact.1.1.14942

12. Sechter I, Mestre F, Hansen DS. Twenty-three years of Klebsiella phage typing: a review of phage typing of 12 clusters of nosocomial infections, and a comparison of phage typing with $\mathrm{K}$ serotyping. Clin Microbiol Infect 2000; 6:233-8; PMID:11168118; http://dx.doi.org/10.1046/j.14690691.2000.00070.x

13. Buckling A, Rainey PB. Antagonistic coevolution between a bacterium and a bacteriophage. Proc Biol Sci 2002; 269:931-6; PMID:12028776; http:// dx.doi.org/10.1098/rspb.2001.1945

14. Marston MF, Pierciey FJ Jr., Shepard A, Gearin G, Qi J, Yandava C, et al. Rapid diversification of coevolving marine Synechococcus and a virus. Proc Natl Acad Sci U S A 2012; 109:4544-9; PMID:22388749; http://dx.doi.org/10.1073/pnas.1120310109

15. Pal C, Maciá MD, Oliver A, Schachar I, Buckling A. Coevolution with viruses drives the evolution of bacterial mutation rates. Nature 2007; 450:107981; PMID:18059461; http://dx.doi.org/10.1038/ nature 06350

16. Dennehy JJ. What Can Phages Tell Us about Host-Pathogen Coevolution? Int J Evol Biol 2012; 2012:396165; PMID:23213618; http://dx.doi. org/10.1155/2012/396165
17. Capparelli R, Nocerino N, Iannaccone M, Ercolini D, Parlato M, Chiara M, et al. Bacteriophage therapy of Salmonella enterica: a fresh appraisal of bacteriophage therapy. J Infect Dis 2010; 201:52-61; PMID:19929381; http://dx.doi.org/10.1086/648478

18. Kashiwagi A, Yomo T. Ongoing phenotypic and genomic changes in experimental coevolution of RNA bacteriophage $Q \beta$ and Escherichia coli. PLoS Genet 2011; 7:e1002188; PMID:21829387; http:// dx.doi.org/10.1371/journal.pgen.1002188

19. Paterson S, Vogwill T, Buckling A, Benmayor R, Spiers AJ, Thomson NR, et al. Antagonistic coevolution accelerates molecular evolution. Nature 2010; 464:275-8; PMID:20182425; http://dx.doi. org $/ 10.1038 /$ nature 08798

20. Lenski RE, Levin BR. Constraints on the Coevolution of Bacteria and Virulent Phage: A Model, Some Experiments, and Predictions for Natural Communities. Am Nat 1985; 125:585-602; http://dx.doi.org/10.1086/284364

21. Gómez P, Buckling A. Bacteria-phage antagonistic coevolution in soil. Science 2011; 332:106-9; PMID:21454789; http://dx.doi.org/10.1126/science. 1198767

22. Meyer JR, Agrawal AA, Quick RT, Dobias DT, Schneider D, Lenski RE. Parallel changes in host resistance to viral infection during 45,000 generations of relaxed selection. Evolution 2010; 64:302434; PMID:20550574

23. Inal JM. Phage therapy: a reappraisal of bacteriophages as antibiotics. Arch Immunol Ther Exp (Warsz) 2003; 51:237-44; PMID:12956433

24. Capparelli R, Nocerino N, Lanzetta R, Silipo A, Amoresano A, Giangrande C, et al. Bacteriophageresistant Staphylococcus aureus mutant confers broad immunity against staphylococcal infection in mice. PLoS One 2010; 5:e11720; PMID:20661301; http:// dx.doi.org/10.1371/journal.pone.0011720

25. Filippov AA, Sergueev KV, He Y, Huang XZ, Gnade BT, Mueller AJ, et al. Bacteriophage-resistant mutants in Yersinia pestis: identification of phage receptors and attenuation for mice. PLoS One 2011; 6:e25486; PMID:21980477; http://dx.doi.org/10.1371/journal. pone.0025486

26. Wei Y, Ocampo P, Levin BR. An experimental study of the population and evolutionary dynamics of Vibrio cholerae $\mathrm{O} 1$ and the bacteriophage JSF4. Proc Biol Sci 2010; 277:3247-54; PMID:20538647; http://dx.doi.org/10.1098/rspb.2010.0651

27. Lythgoe KA, Chao L. Mechanisms of coexistence of a bacteria and a bacteriophage in a spatially homogeneous environment. Ecol Lett 2003; 6:326-34; http:// dx.doi.org/10.1046/j.1461-0248.2003.00433.x

28. Flores CO, Meyer JR, Valverde S, Farr L, Weitz JS. Statistical structure of host-phage interactions. Proc Natl Acad Sci U S A 2011; 108:E288-97; PMID:21709225; http://dx.doi.org/10.1073/ pnas. 1101595108

29. Wolf A, Wiese J, Jost G, Witzel KP. Wide geographic distribution of bacteriophages that lyse the same indigenous freshwater isolate (Sphingomonas sp. strain B18). Appl Environ Microbiol 2003; 69:23958; PMID:12676728; http://dx.doi.org/10.1128/ AEM.69.4.2395-2398.2003

30. Atanasova NS, Roine E, Oren A, Bamford DH, Oksanen HM. Global network of specific virus-host interactions in hypersaline environments. Environ Microbiol 2012; 14:426-40; PMID:22003883; http://dx.doi.org/10.1111/j.1462-2920.2011.02603.x

31. Sulakvelidze A, Alavidze Z, Morris JG Jr. Bacteriophage therapy. Antimicrob Agents Chemother 2001; 45:649-59; PMID:11181338; http://dx.doi.org/10.1128/AAC.45.3.649-659.2001 